\title{
THE EFFECT OF THE ADSORPTION UPON THE SELECTIVITY AND THE EFFECTIVENESS FACTOR ${ }^{*}$
}

\author{
HIROSHI KOMIYAMA AND HAKUAI INOUE \\ Department of Chemical Engineering, the Faculty \\ of Engineering, University of Tokyo
}

\begin{abstract}
The effectiveness factor and the selectivity are calculated for a consecutive reaction where the kinetics follow the Langmuir-Hinshelwood types of rate equations. The computational results are extended to cases where the first order specific catalytic activity varies inside a catalyst pellet and it is shown that the selectivity is greatly changeable by the difference in the distribution of the rate constant inside the catalyst even in the case where the overall reaction rate of the reactant has the same value.
\end{abstract}

In the previous work ${ }^{2)}$ on the hydrogenation of acetylene on nickel catalyst, the authors found that the selectivity to ethylene was reduced with increase in catalyst size, and the degree of the reduction in selectivity was larger than that predicted for a first order consecutive reactions system as derived by Wheeler ${ }^{3)}$. In that paper ${ }^{2}$, the discrepancy was proved to be due to the difference in kinetics which followed the Langmuir-Hinshelwood types of rate equations in our experiments.

In this paper, the effects of the types of kinetics upon the selectivity of the intermediate product and upon the effectiveness factor for the rate of conversion of the reactant are discussed.

The systems assumed in the analysis are as follows: the catalyst is a porous spherical particle, the reaction is consecutive and is

$$
A \stackrel{R_{1}}{\longrightarrow} B \stackrel{R_{2}}{\longrightarrow} C
$$

and the effective diffusion coefficients of the components $A, B$ and $C$ are identical.

Basic equations and boundary conditions are expressed by Eqs. (1) and (2).

$$
\begin{aligned}
& D\left(\frac{d^{2} p_{A}}{d r^{2}}+\frac{2}{r} \frac{d p_{A}}{d r}\right)=R_{1} ; \\
& D\left(\frac{d^{2} p_{B}}{d r^{2}}+\frac{2}{r} \frac{d p_{B}}{d r}\right)=-R_{1}+R_{2} \\
& \text { At } r=R, \quad p_{A}=p_{A s} \text { and } p_{B}=p_{B s} ; \\
& \text { at } \quad r=0, \quad \frac{d p_{A}}{d r}=\frac{d p_{B}}{d r}=0 .
\end{aligned}
$$

For the system, four types of reaction kinetics are analyzed which are shown in Table 1. For comparison with the previous experiments, where $K_{A} p_{A s}=23$ and $s=7$, the conditions for computations are taken for all

* Received on June 23, 1969

The annual meeting of the Society of Chemical Engineers, Japan (at Nagoya, 1968)

** Stored in the Soc. of Chem. Engrs., Japan. (1 copy 100 Yen) systems as

$$
\begin{aligned}
& p_{B s}=0, \quad K_{i p_{A s}}=0,10,30 \\
& M_{1}=0.5 \sim 50 \text { and } s=7 .
\end{aligned}
$$

The condition of $p_{B s} \doteqdot 0$ is realized in a flow reactor with short contacting time. The selectively under the condition of $p_{B s}=0$ can be attained by extrapolation of the data obtained with various contacting times as described in the previous paper ${ }^{2}$.

\section{Computational Results}

1) Effectiveness factor $\left(\boldsymbol{E}_{1}\right)$

The computational results for some of the conditions are shown in Fig. 1. Here, the moduli for the catalyst are expressed by the use of the rate at the outer surface of the catalyst, as shown in Table 1 . The values of $E_{1}$ for the values of $M_{1}$ larger than about 20 can be derived theoretically in Case 1 and Case 2.

$$
\text { Case } 1 \quad E_{1}=3 / M_{1}
$$

Case $2^{* *} E_{1}=$

$$
3 / M_{1} \frac{\sqrt{2\left(1+K_{A} p_{A s}\right)\left(\overline{K_{A} p_{A s}-}-\ln \left[1+K_{A} p_{A s}\right]\right)}}{K_{A} p_{A S}}
$$

\begin{tabular}{|c|c|c|c|c|}
\hline \multicolumn{5}{|c|}{ Table 1} \\
\hline \multicolumn{2}{|c|}{$\begin{array}{l}\text { Adsorbed } \\
\text { component }\end{array}$} & \multicolumn{2}{|c|}{ Rate expression } & Modulus : $M_{1}$ \\
\hline Case 1 & & $R_{1}=k_{3} p_{A}$ & $R_{2}=k_{2} p_{B}$ & $R \sqrt{\frac{k_{1}}{D}}$ \\
\hline Case 2 & $\mathrm{~A}$ & $R_{1}=\frac{k_{1} p_{A}}{1+K_{A} p_{A}}$ & $R_{2}=\frac{k_{2} p_{B}}{1+K_{A p_{A}}}$ & \\
\hline & & & $R \sqrt{-}$ & $\frac{k_{1}}{D\left(1+K_{A p_{A s}}\right)}$ \\
\hline Case 3 & $\mathrm{~B}$ & $R_{1}=\frac{k_{1} p_{A}}{1+\bar{K}_{B p_{B}}}$ & $R_{2}=\frac{k_{2} p_{B}}{1+K_{B p_{B}}}$ & $R \sqrt{\frac{k_{1}}{D}}$ \\
\hline Case 4 & $\mathrm{C}$ & $R_{1}=\frac{k_{1} p_{A}}{1+K_{C p_{C}}}$ & $R_{2}=\frac{k_{2} p_{B}}{1+K_{C p_{C}}}$ & $R \sqrt{\frac{k_{1}}{D}}$ \\
\hline
\end{tabular}

These relations are shown by the dotted lines $A, B_{1}$ and $B_{2}$ in Fig. 1 and, as expected, coincide well with the computational results.

2) Selectivity $(S)$

In the simple consecutive reaction, the selectivity to 


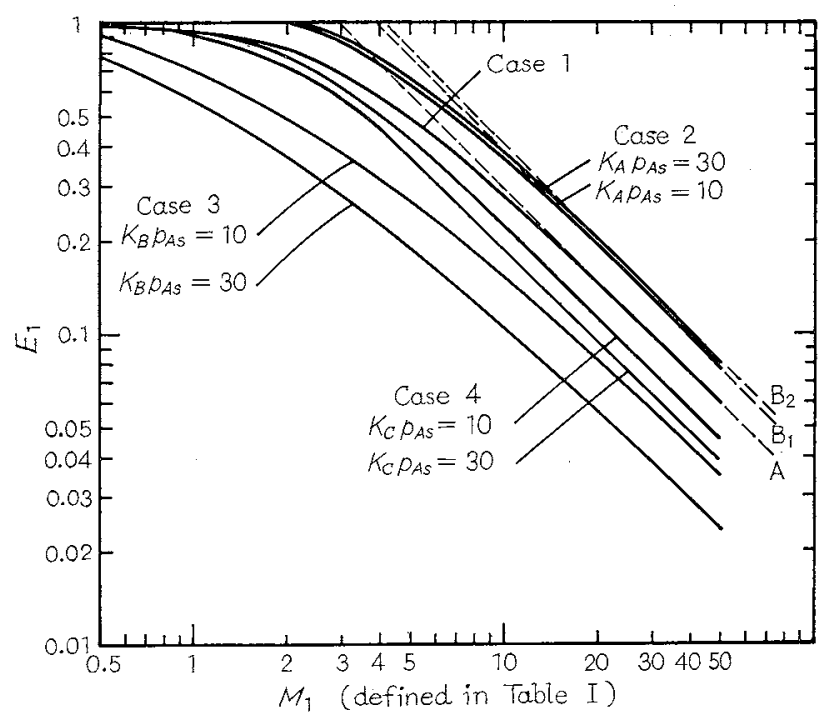

Fig. I The relations between effectiveness factor $\left(E_{1}\right)$ and $M_{1}(s=7)$

the intermediate product becomes to unity as $M_{1}$ becomes smaller than unity and decreases with increase of $M_{1}$. The detailed relations of the selectivity with the modulus for four cases are shown in Fig. 2. As shown in the figure, the selectivity is lowest in Case 2. Case 3 and 4 give a larger selectivity than Case 1 . In the region where $M_{1}$ is not so large, Case 3 gives a larger selectivity than Case 4.

The limiting selectivity at large $M_{1}$ and $K_{A} p_{A s}$ in Case 2 can be derived theoretically and the result is ** :

$$
S=\frac{s}{s-1} \cdot \frac{(3-\sqrt{(1+8 / s)})}{4}
$$

Along with these computations, the concentration and the reaction rate profiles inside the particle are also evaluated. These results are applicable to the systems where the apparent rate constant is distributed inside the catalyst. For example, these systems are equivalent to the cases where the first order catalytic rate constant $\left(k_{a p p .}\right)$ changes as $k_{1 \text { (ox 2)a ap }}=\frac{k_{1 \text { (or } 2)}}{1+K_{i} p_{i}(r)}$. Here $p_{i}(r)$ is the partial pressure distribution of $i$ component inside the particle, which is obtained in the above computation.

That is :

Case $1 \quad k_{a p p}$. is constant.

Case $2 k_{a p p}$. increases towards the center of the catalyst.

Case $3 \quad k_{a p p}$. decreases towards the center of the catalyst at small $M_{1}$ and has the minimum on an intermediate position at large $M_{1}$.

Case $4 k_{a p p}$. decreases towards the center of the catalyst.

From this analysis, it is clear that the selectivity is greatly changeable by the difference of the distributions

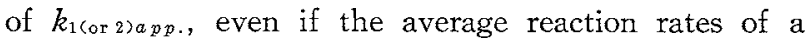
single catalyst pellet $\left(k_{1 a p p}\right.$. (at surface) $\left.E_{1} p_{A s}\right)$ are the same. This paper is the first one which shows the large effect of the distribution of the apparent rate

** Stored in The Soc. of Chem. Engrs., Japan. ( 1 copy 100 Yen)

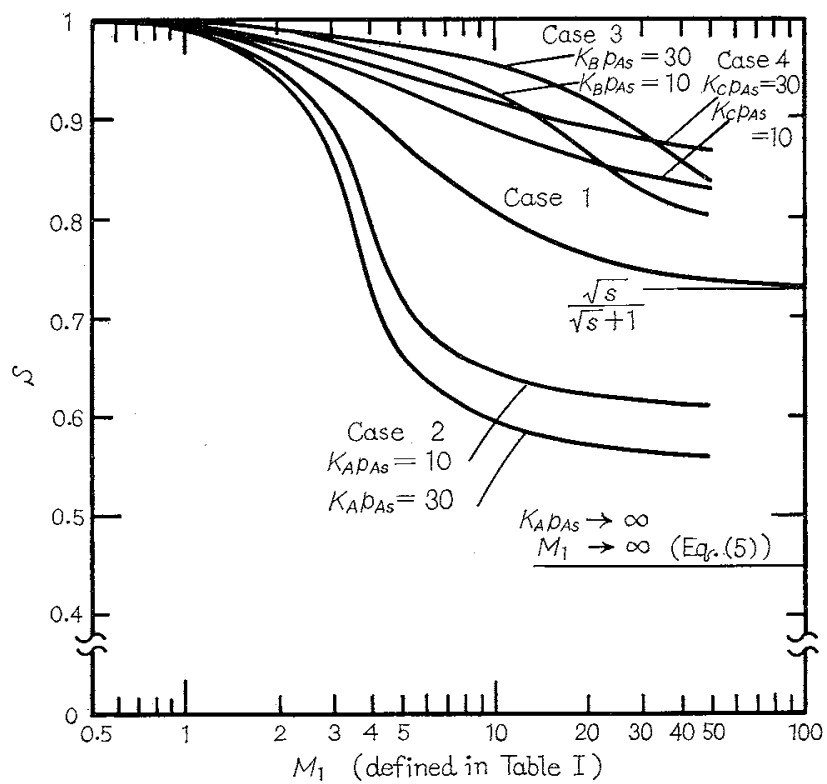

Fig. 2 The relations between the selectivity (S) and $M_{1}(s=7)$

constant inside a catalyst particle upon the selectivity, with the exception that J. B. But ${ }^{13}$ calculated the selectivity for the nonisothermal first order reaction system.

This treatment may be applied to the analysis of the gradual deactivation of a single catalyst particle. The observed behaviour of the selectivity and the effectiveness factor during the reaction will give insight into the mechanism of the deactivation of the catalyst. Details of the studies of catalyst deactivation will be presented in the near future.

\section{Nomenclature}

$A \quad=$ reactant

$B \quad=$ intermediate product

$C \quad=$ final product

$D \quad=$ effective diffusion coefficient inside the catalyst particle

$E_{1} \quad=$ effectiveness factor for the rate of conversion of the reactant

$k_{1(\text { or } 2)}=$ rate constant defined in Table 1

$k_{1 \text { (or 2)app. }}=$ apparent rate constant

$K_{i} \quad=$ equilibrium constant of adsorption of $i$ component ; $i=A, B, C$

$M_{1} \quad=$ modulus defined in Table 1

$p_{i} \quad=$ partial pressure of $i$ component

$p_{i s} \quad=$ partial pressure of $i$ component at the outer surface of a catalyst particle; $i=A, B, C$

$r \quad=$ radial distance in a catalyst particle

$R \quad=$ radius of the catalyst particle

$R_{\text {I (or 2) }}=$ rate of the first (or second) step of the consecutive reactions system

$s \quad=k_{1} / k_{2}$

$S \quad=$ selectivity defined as the ratio of $B$ produced to $A$ consumed

\section{Literature Cited}

1) Butt, J. B.: Chem. Eng. Sci., 21, 275 (1966)

2) Komiyama, H., Inoue, H.: J. Chem. Eng. Japan, 1, 142 (1968)

3) Wheeler, A.: Catalysis, Ed. by Emett, P. H., Vol. II, 105 (1955) 